\title{
BMJ Open Optimising patient active role with a user-centred eHealth platform (CONCERTO+) in chronic diseases management: a study protocol for a pilot cluster randomised controlled trial
}

To cite: Gagnon M-P, Ndiaye MA, Larouche A, et al. Optimising patient active role with a user-centred eHealth platform (CONCERTO+) in chronic diseases management: a study protocol for a pilot cluster randomised controlled trial. BMJ Open 2019;9:e028554. doi:10.1136/ bmjopen-2018-028554

- Prepublication history and additional material for this paper are available online. To view these files, please visit the journal online (http://dx.doi. org/10.1136/bmjopen-2018028554).

Received 14 December 2018 Revised 14 January 2019 Accepted 16 January 2019

D Check for updates

(c) Author(s) (or their employer(s)) 2019. Re-use permitted under CC BY-NC. No commercial re-use. See rights and permissions. Published by BMJ.

For numbered affiliations see end of article.

\section{Correspondence to}

Professor Marie-Pierre Gagnon; marie-pierre.gagnon@fsi. ulaval.ca

\section{ABSTRACT}

Introduction Multimorbidity increases care needs and primary care use among people with chronic diseases. The Concerto Health Program (CHP) has been developed to optimise chronic disease management in primary care services. However, in its current version, the CHP primarily targets clinicians and does not aim to answer directly patients' and their informal caregivers' needs for chronic disease management. Various studies have shown that interventions that increase patient activation level are associated with better health outcomes. Furthermore, educational tools must be adapted to patients and caregivers in terms of health literacy and usability. This project aims to develop, implement and evaluate a usercentred, multifunctional and personalised eHealth platform (CONCERTO+) to promote a more active patient role in chronic disease management and decision-making.

Methods and analysis This project uses a collaborative research approach, aiming at the personalisation of CHP through three phases: (1) the development of one module of an eHealth platform based on scientific evidence and user-centred design; (2) a feasibility study of CONCERTO+ through a pilot cluster randomised controlled trial where patients with chronic diseases from a primary healthcare practice will receive CONCERTO+ during 6 months and be compared to patients from a control practice receiving usual care and (3) an analysis of CONCERTO+ potential for scaling up. To do so, we will conduct two focus groups with patients and informal caregivers and individual interviews with health professionals at the two study sites, as well as health care managers, information officers and representatives of the Ministry of Health.

Ethics and dissemination This study received ethical approval from Ethics Committee of Université Laval. The findings will be used to inform the effectiveness of CONCERTO+ to improve management care in chronic diseases. We will disseminate findings through presentations in scientific conferences and publication in peer-reviewed journals.

Trial registration number NCT03628963; Pre-results.
Strengths and limitations of this study

The design of a user-centred technological solution is adapted to the needs of patients with chronic diseases and their literacy level.

- The inclusion of informal caregivers in the use of CONCERTO+ is a novelty.

- The pilot test will provide data for feasibility, acceptability and usefulness of CONCERTO+.

- Good potential for sustainability given that it will be implemented in the real context of primary care practice with the collaboration of clinical teams.

- As a limitation, this project seems ambitious for its entire achievement in 2 years.

\section{INTRODUCTION}

\section{Background}

Chronic diseases are the number one cause of mortality in the world and account for nearly $70 \%$ of deaths. ${ }^{1}$ In Canada and around the world, multimorbidity, which means people who have more than two chronic diseases, is increasing. ${ }^{2}$ In addition to often making life more difficult for people living with these conditions, the rise in multimorbidity is putting pressure on the Canadian healthcare system and causing overuse of care and services. ${ }^{3}$ In the province of Quebec, $45 \%$ of people aged 20 and over have more than two chronic diseases, ${ }^{4}$ and $80 \%$ of chronic disease consultations are done in primary healthcare services. ${ }^{5}$ Multimorbidity increases care needs as well as the complexity of healthcare services required in primary care, especially when it comes to applying recommendations for good clinical practices. ${ }^{5}$ The total cost of the six most common chronic diseases in 
Quebec (ischaemic heart disease, cerebrovascular disease, chronic obstructive pulmonary disease, cancer, hypertension and diabetes) has been estimated at \$C8.1 billion, and this may rise up to $\$ \mathrm{C} 13$ in 2030 if no substantial change is made. ${ }^{6}$

In Quebec, primary care services have the main responsibility to support people with chronic diseases and their informal caregivers, jointly with other stakeholders of the local health network. ${ }^{78}$ However, primary care services suffer from many challenges and organisational constraints, in particular, the difficulty of accesswith a large proportion of Quebeckers without a family doctor-and the wait times that are among the longest in Canada. ${ }^{9}{ }^{10}$ Furthermore, the fragmentation of healthcare processes and the gaps in information transfer are recognised sources of inefficiency that make critical the integration and continuity of care for chronic diseases. ${ }^{711}$ To overcome these issues, many approaches linking healthcare providers, patients, caregivers and the organisation of healthcare services are promoted. ${ }^{12}$ The central role of patients in the management of their disease, which depends on their active involvement, is recognised as a key component in chronic disease management. ${ }^{13}$

Active patient involvement requires that patients have the knowledge, skills and self-confidence to manage their health and healthcare. ${ }^{14}$ Various studies have shown that interventions increasing patient activation level are associated with better health outcomes ${ }^{15-22}$ and decreased costs. ${ }^{23}$ However, active patient involvement and the quality of the interactions with health providers will partially depend on patient's knowledge of the disease and the needed care, in addition to their interpersonal skills as well as their ability to communicate their expectations, needs and preferences to their healthcare team. ${ }^{245}$ It is, therefore, important to offer patients and caregivers relevant information adapted to their health literacy level. According the following definition, 'Health literacy is linked to literacy and entails people's knowledge, motivation and competences to access, understand, appraise and apply health information in order to make judgements and take decisions in everyday life concerning healthcare, disease prevention and health promotion to maintain or improve quality of life during the life course'. ${ }^{26}$ For their part, health professionals must also have the communicational and interpersonal skills required to work in a team and share information appropriately with patients in order to support their active involvement. ${ }^{24}$ Thus, it becomes important to act in advance by supporting patients' autonomy and involvement in the care dynamic, and by promoting informational and educational relationships in disease management. ${ }^{25-27}$ Therefore, it is crucial that information and educational tools are adapted to patients and caregivers in terms of literacy level and presentation. ${ }^{28-30}$

eHealth technologies offer a potential to support chronic disease management. Some studies have shown positive effects on clinical processes (better adherence to care protocols, reduced errors and improved monitoring and callback rates), on quality of care and effectiveness, and on patient outcomes. ${ }^{31-36}$

Systematic reviews support the role of electronic personal health records and electronic portals allowing patient access to their health records in order to promote their active participation in their care. ${ }^{37-39}$ However, to achieve expected outcomes, eHealth technologies should first be adopted and used in an appropriate manner by patients and health professionals. ${ }^{40}$ Therefore, end-user involvement in the development of eHealth solutions is an imperative. ${ }^{41}$ Moreover, eHealth literacy, which is inspired by the health literacy concept but focuses specifically on optimal eHealth solutions use, should be considered in order to ensure that the solutions are adapted to the capabilities of targeted users. ${ }^{3042}$ While the number of eHealth solutions continues to increase, with more than 325000 mobile health applications in $2017,{ }^{43}$ the majority of them (53\%) are used by less than 5000 people and are often abandoned after a short trial period. ${ }^{44-46}$ User involvement-including patients, informal caregivers and health professionals - is identified to be among the conditions to ensure that eHealth solutions have a real impact. Thus, all these stakeholders must be involved throughout the different stages of technology development, from conception to assessment. ${ }^{47}$ Based on efficient chronic care models, high-potential technologies and patient involvement as active partner of their care, we suggest to develop an innovative and mobilising project in order to improve patient care and experience.

\section{METHODS AND ANALYSIS}

The following methods adhere to the Standard Protocol Items: Recommendations for Interventional Trials guidelines for the reporting of study protocols.

This project is a collaborative work involving IT developers from Concerto Health Program (CHP), designers, clinicians, technological partners and patient representatives. The aim is to develop, implement and evaluate a module of a multifunctional and personalised eHealth platform, CONCERTO+, through a pilot study for optimising patient active role in medical follow-up, decision-making, satisfaction towards healthcare services and quality of life. The specific objectives are to: (1) develop a module of a multifunctional and personalised eHealth platform integrated to the CHP for patients and caregivers allowing them to engage in the follow-up and management of their chronic diseases; (2) test the integration of CONCERTO+ in monitoring care pathways of three frequent coexisting chronic diseases (diabetes, hypertension, dyslipidaemia) and assess the usefulness and acceptability of the solution for patients with chronic diseases and their caregivers and (3) assess the scalability of the CONCERTO+ solution.

Phase 1: development of the eHealth solution module We will conduct a rapid literature review on the effects of eHealth interventions for supporting active 
involvement of patients with chronic diseases in their primary care team. For this purpose, we will follow the rapid review method suggested by Lawani $e t a l^{48}$ and consider the latest evidence on eHealth interventions for chronic diseases monitoring and care. We will consider the following 'Problem, Intervention, Comparison, Outcomes' elements: (P): three targeted chronic diseases (diabetes, high blood pressure, dyslipidaemia), alone or combined; (I) all eHealth interventions implemented in primary care and that directly involve patients (eg, electronic medical records (EMRs), patient diary, patient portal, specific computerised monitoring for a chronic disease and technological interventions focused on lifestyle modifications; $(\mathrm{C})$ : routine follow-up; $(\mathrm{O})$ : health outcomes specific to the disease (eg, glycosylated hemoglobin (HbA1c) for diabetes), generic health outcomes (eg, mortality, quality of life), patient outcomes (eg, involvement, personal efficacy) and practices and process outcomes (eg, test numbers, emergency visits, hospitalisations). First, we will start to consult existing systematic reviews, in particular, that of Irizarry et al, ${ }^{38}$ and a review of reviews that we have already completed. ${ }^{49}$ We will also document issues relating to needs, expectations and concerns in terms of eHealth solutions for patients, their informal caregivers and healthcare providers. This information will provide evidence summaries describing each eHealth solution associated with each targeted health issues, as well as information on the risks and benefits of these solutions. We will then use the methods suggested by Giguere et $a b^{\tilde{D}^{0}}$ to develop decision boxes to involve patients and their informal caregivers in the choice of functionalities and contents to develop in the CONCERTO+ solution, in line with an integrated care system (figure 1).

A first prototype will be developed by the design and technology teams, in close collaboration with researchers, health professionals and patient representatives who will identify the functionalities to include in the CONCERTO+ solution. Given the time limit of the project, we will classify the required functionalities in three types: (1) essential and priority, (2) important but not priority and (3) required in the future.

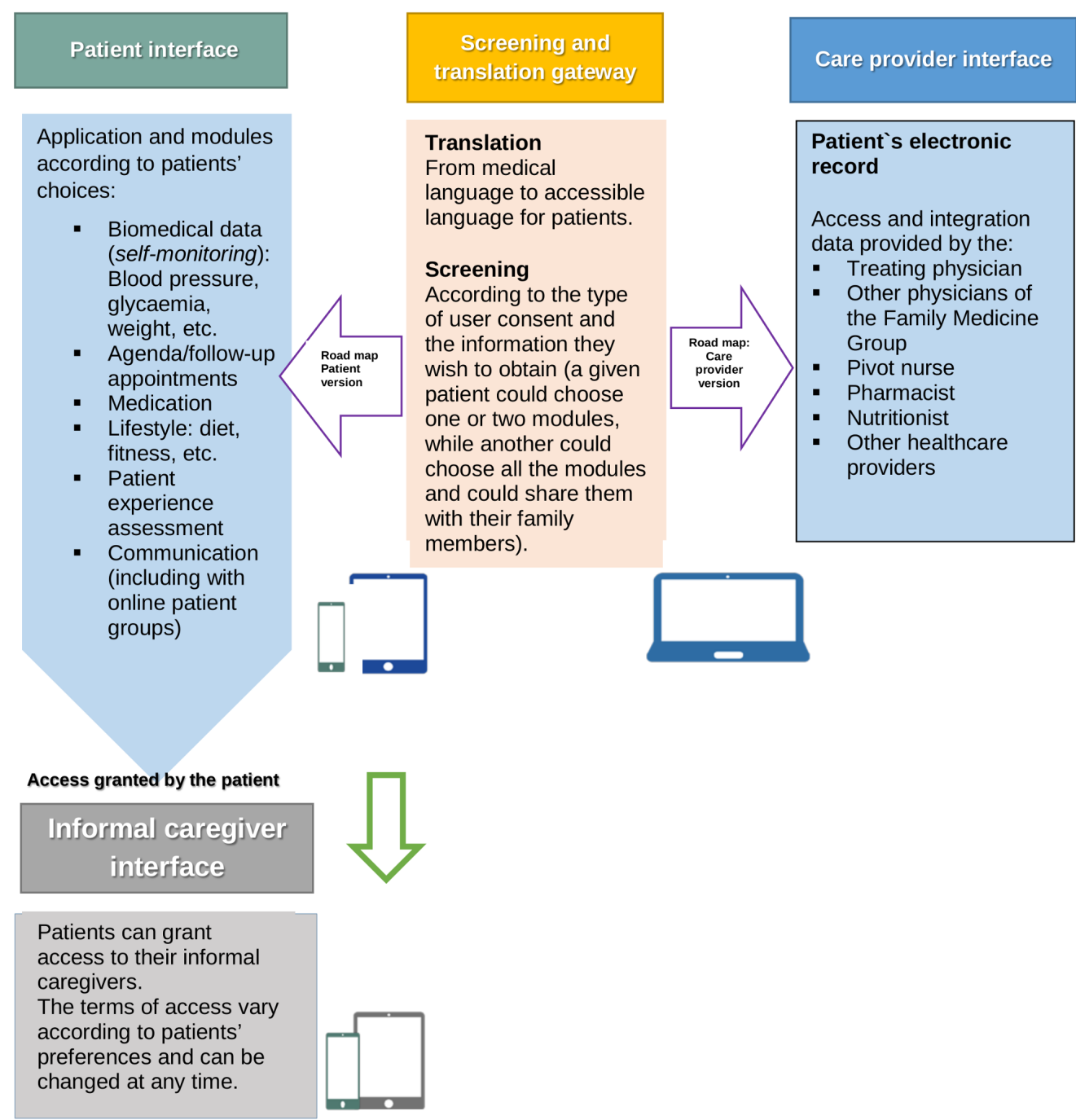

Figure 1 Embedded healthcare system. 
For the development of the eHealth platform module, a user-centred approach will be used, based on three cycles with users. Iterative testing sessions will take place at the usability laboratory of Université Laval (UL) lead by HOW, providing all the equipment needed to conduct usability studies. Students in graphical and interaction design, under the direction of three experts from the School of Design of UL (EK, FLép, JR), will participate in the development of the platform's visual environment. An expert in eHealth literacy (CD) will ensure that contents of the clinical monitoring tools already integrated in the CHP are adapted to a general audience according to recommendations of the health literacy guide ${ }^{51}$ in addition of tools that provide understandable information (eg, link to a popular glossary of medical terms: https://publications.santemontreal.qc. ca/uploads/tx_asssmpublications/litteratie_v9.pdf).

The integration of the CONCERTO+ solution with the CHP will be ensured by the Concerto Health Group team who will work closely with the designers and researchers. Health professionals in primary care services from the sites participating in the pilot project will also be consulted to validate the match between the CONCERTO+ solution and care pathways for professionals offered by the CHP.

\section{Patient and public involvement}

A patient partner (informal caregiver) is involved as research partner at key stages of the study. His experience in caring of a patient with diabetes informed us on needs of patients, research focus, methods for collecting data for the study and dissemination strategy through patient and citizen groups associations.

Our patient partner is invited at each research team meeting to make sure that the research questions are aligned with patients' needs. He gives his input in refining the focus of the research questions. He made valuable contributions in the design of the study.

In the first step of the study, the development of the first prototype, our patient partner helped us to recruit patients by sharing the invitation through his personal contacts and network and gave feedback for the positive and negative points cons of the prototype development. He was also invited to contribute in editing the paper and is considered as a coauthor.

To develop our dissemination strategy, we will review the results with the patient partner and integrate his feedback to ensure that we presented the results in the most effective way for the general populations. We will send a summary of the research results to study participants who have provided their mailing address in the consent form and we will also organise events for patients and citizen groups and associations, such as outreach communications and scientific café.

In this study, participants will assess the burden of the intervention by participating in focus groups.

\section{Phase 2: pilot cluster randomised controlled trial}

The phase 2 of the project will consist of a feasibility study based on a pilot cluster randomised controlled trial
(c-RCT). Given the nature of the intervention, patients with chronic diseases are followed by a small team of primary care clinicians.

\section{Study setting}

The study will be conducted in two family medicine groups (FMGs) from the same health region (in the province of Quebec) but covering distinct areas, they have been selected as the clusters.

\section{Eligibility criteria}

Patients with two or more targeted chronic diseases (diabetes, hypertension, dyslipidaemia) and who had three or more visits in the last 12 months will be eligible. Adults whose legal incompetence has been established by a court are not eligible.

\section{Intervention}

The intervention is the device CONCERTO+, a user-centred, multifunctional and personalised eHealth platform. Both groups, experimental and control, have the same criteria with respect to participant eligibility. Experimental group from FMG 1 will use CONCERTO+ application during 6 months. Control group from FMG 2 will not use the application CONCERTO+ but continue to receive usual care. The objective is to assess the feasibility, acceptability and potential effectiveness of the device CONCERTO+.

\section{Outcomes}

Patient involvement in their care following the use of CONCERTO+ will be our primary outcome of interest. We will use Patient Activation Measure $(\mathrm{PAM})^{52}$ which is built on patient knowledge, skills and confidence that are directly targeted by the intervention.

The score of the activation level obtained (between 0 and 100) shows the degree of ability to manage their health with confidence according to the following scale ranges: strongly disagree $=1$; disagree $=2$; agree $=3$; strongly agree $=4$. Patients with a higher activation level are likely to have better health outcomes. Patients answer to a survey of 13 questions with the following scoring for each answer:

1. Not believing that activation is important $(\leq 47)$.

2. Lack of knowledge or confidence to take action (47.155.1).

3. Beginning to take action (55.2-67).

4. Taking action $(\geq 67.1)$.

The PAM 13 questionnaire has been validated in French. We will ask a licence to use, which is free for up to 250 patients in an academic research context. ${ }^{53}$ The survey will be completed by participants of the two groups at baseline and 6 months later. This period of CONCERTO+ use is enough to achieve the intended outcomes.

Secondary outcomes of interest are: (1) Impacts of CONCERTO+ use on process indicators and care outcomes, measured with questions adapted from Glasgow et $a \tilde{l}^{4}$ and validated in the previous CHP assessment. To 
Table 1 Distribution of outcomes measures through time

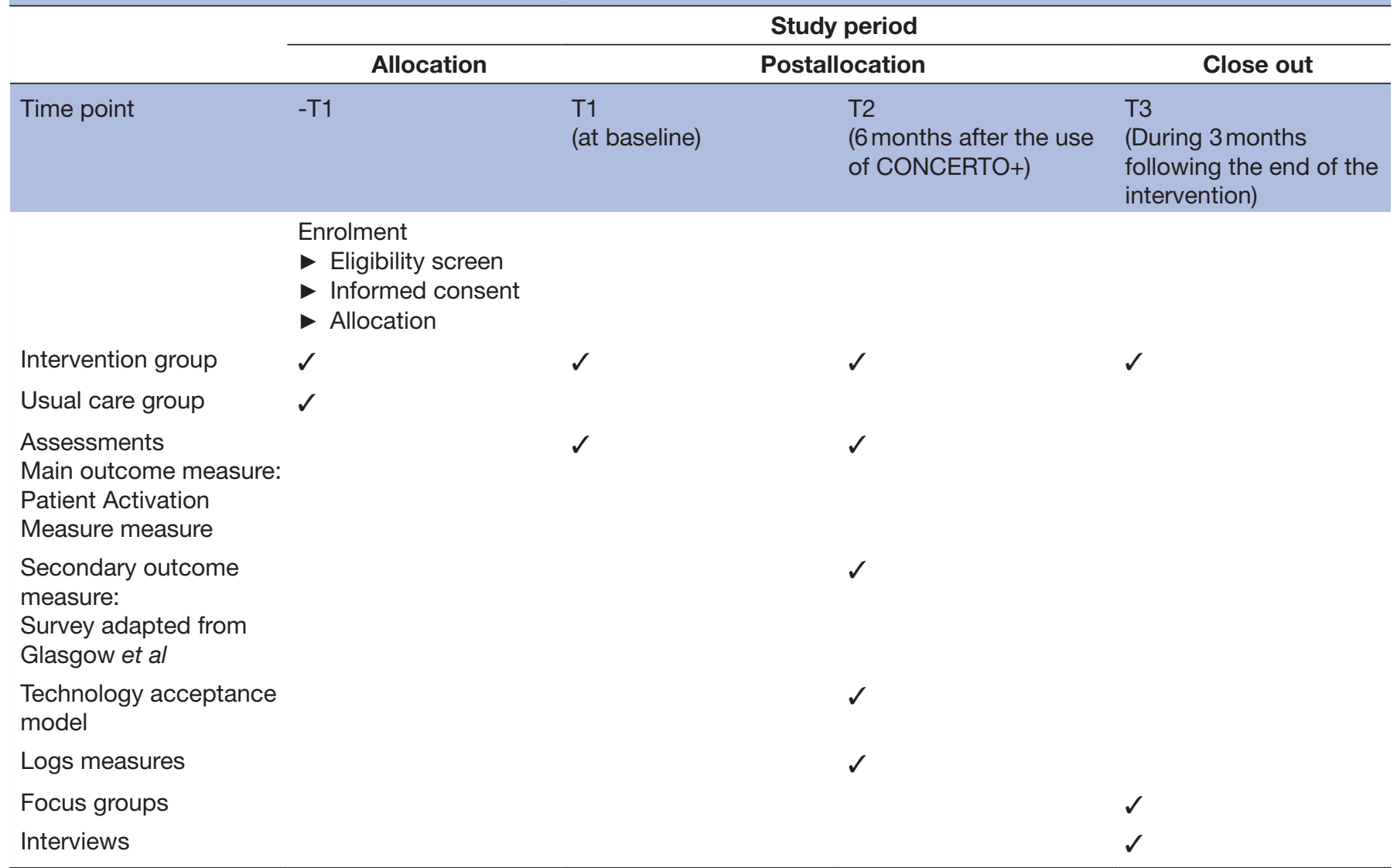

measure these outcomes, patients will answer to a questionnaire after 6 months use of CONCERTO+. This questionnaire comprises five scales based on the key components of CONCERTO+ and covering the following dimensions: solving-problems/advices, delivery system design/decision support, goal setting/tailoring, follow-up/coordination, overall care. Items are scored on a 5-point scale with the following values: (1) (almost never); (2) (generally not); (3) (sometimes); (4) (most of the time); (5) (almost always). For each scale, higher scores are expected to be associated with better care outcomes.

(2) The acceptability of the device CONCERTO+ will be assessed by patients and informal caregivers, at the end of the intervention with:

1. A short survey adapted from the technology acceptance model ${ }^{55}$ that includes three criteria (perceived ease of use, perceived usefulness, behavioural intention to use) with the following scoring: strongly disagree $=1$; disagree $=2$; agree $=3$; strongly agree $=4$. Higher scores indicate a better acceptance of the use of CONCERTO+.

2. The use of CONCERTO+ that will be measured by logs (numbers of tests ordered, emergency visits and hospitalisations) (see online supplementary files 1 and 2).

\section{Participant timeline}

Table 1 shows the distribution of outcomes measures through time. The first survey will be completed at baseline and 6 months after the use of CONCERTO+, in order to see the effects of the use of CONCERTO+ during the process of care. The second survey will be completed 6 months after in order to assess the effects of the use of CONCERTO+, and the third survey will be completed by patients and informal caregivers after the intervention in order to assess its acceptability.

\section{Sample size calculation}

Based on a similar study, ${ }^{56}$ a sample of 200 patients is enough to detect a difference of 2 points on the PAM score, with a power of $90 \%$ and an alpha of 0.05 . Indeed, the assessment of online education intervention to patients with chronic disease showed a significant difference of 6 points on the PAM score in the experimental group $(n=58)$, whereas the difference was not significant in the control group $(n=68) .{ }^{56}$ Such a difference may be considered clinically significant because each additional point on the PAM score is associated with a $2 \%$ decrease in hospitalisations. ${ }^{53}$ Considering an attrition rate of $15 \%$, the sample size should remain relevant to detect a difference of at least 2 points on the PAM score, as differences reported in similar studies range from 2.5 to 6.5 points. ${ }^{14}$

\section{Recruitment strategy}

For the phase 1, the development of the eHealth solution module, we will recruit 7-10 patients and informal caregivers from convenience samples of volunteers joined 
through patient associations and mailing lists of our institution (UL). Eligible individuals will meet the following criteria: (1) have one or more targeted chronic diseases (diabetes, hypertension, dyslipidaemia); (2) had three or more medical visits in the last 12 months; (3) are 18 years old and over; (4) reside in the greater Québec area; (5) have an interest in technology; (6) are able to speak and read in French and (7) are available to participate in three validation sessions.

For the phase 2, the pilot c-RCT, a note will be added in the EMR of patients who had been preselected, and at their next visit at the FMG, the receptionist will give them an information sheet about the study to invite them to participate. Interested patients will be invited to call the research assistant using a toll-free number or to leave their contact information to the receptionist who will forward them to the research assistant. Then, patients will be contacted by the research assistant to validate their eligibility and confirm their interest. Recruitment will end when 100 patients are recruited from each site. We will ensure an equal distribution of participants according to their sex, and we will consider specific aspects of patient recruitment, particularly living alone, the presence of dependents and their literacy level. The recruitment chart is presented in figure 2 .

\section{Allocation}

The patient will be selected randomly with the help of the participating FMG by searching the local EMR system. A preselection of patients will be done by the four nurses involved in chronic disease care at the participating FMG. For each site, a sample of 200 patients (see sample size calculation) stratified by sex, age group and a number of chronic diseases, will be randomly preselected by a statistician not involved in the team, using a computerised programme. Then, the statistician will reveal group assignment through a call to the responsible of each FMG in the presence of a research team member.

\section{Blinding}

Given the nature of the intervention, participating patients and healthcare providers cannot be blinded, but the outcome assessor will be blinded to participant assignment.

\section{Phase 3: scaling-Up Potential of the Solution}

For phase 3, the analysis of CONCERTO+ potentialfor scaling-up will be done by documenting factors and conditions associated with the sustainability and scaling-up of the solution. To do so, we will conduct: (1) two focus groups with patients and informal caregivers who participated in the study (one with the experimental group and one with the control group, each group gathering between 8 and 12 participants); (2) semistructured individual interviews with health professionals as well as with healthcare managers, information officers and representatives of the Ministry of Health and Social Services will be conducted at the two study sites two FMG of one region in the province of Quebec). The number of interviews will be determined according to the data saturation principle, but is estimated to be around 15 participants in total. Interviews with patients, informal caregivers and health professionals will include questions about factors facilitating or limiting sustained use of the CONCERTO+ solution by patients and informal caregivers, and the support of this use by health professionals, inspired by a recent study on personal electronic health record. ${ }^{57}{ }^{58}$ Questions for managers and decision-makers will be based on ExpandNet framework ${ }^{59}$ that proposes 12 elements helping to appreciate the potential of innovation expansion at different time of its progress (see online supplementary files 3 and 4 ).

\section{Data analysis plan}

The study started in 2017 and will end in 2019. Data will be collected managed and analysed at each step of the project. For the phase 1, we started to collect data in October 2018; for the phase 2, we will start in April 2019 and the phase 3 will start in November 2019. We will ensure that surveys are correctly completed in order to avoid many missing data. Quantitative data will be analysed using standard statistical tests such as analysis of variance. We will compare the scores for repeated measurements between the two groups, controlling for the initial PAM score. We will also make tests according to sex, literacy level and comorbidity because these variables are associated with the PAM score ${ }^{60}$ Focus groups discussions and interviews will be recorded with participants' consent, and the content will be transcribed verbatim. The qualitative analysis will consist of a thematic-pragmatic content analysis ${ }^{61}$ using the NVivo V.10 software. We will use an inductive-deductive analysis, in an iteratively and flexible way, which allows a hybrid codification from the conceptual dimensions of the model and the emergent themes. ${ }^{62}$ We will verify the role of the identified dimension in the literature as the initial basis for analysis, while remaining open to the advent of other context-specific aspects. Findings from qualitative analyses will be triangulated with quantitative data to see commonalities among participants' characteristics. We will compare intervention and control groups to judge the potential effectiveness of CONCERTO+ using process and care outcomes, and these results will inform the relevance of conducting a definitive trial to assess the effectiveness of CONCERTO+ for improving health outcomes. Participants will also be asked about the usefulness of the CONCERTO+ solution in supporting their disease self-management.

\section{Monitoring}

A data monitoring committee is not required for this study due to low risk of adverse events. The principal investigator has the authority to suspend or terminate the study at any time if any major problem occurs.

\section{Ethics and dissemination}

This study received ethical approval from the Research Ethics Committee of Université Laval; approval number: 


\section{Phase 1}
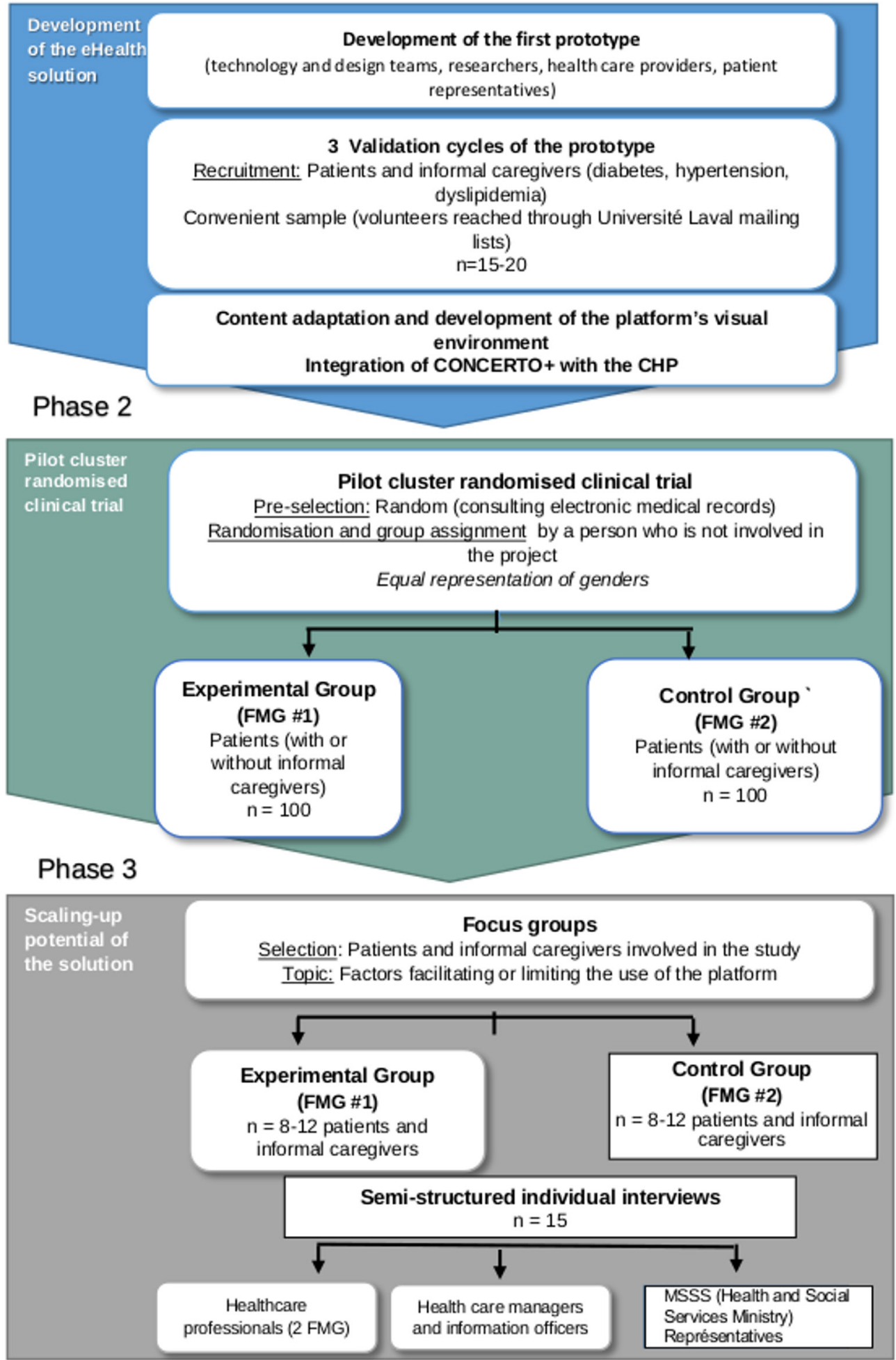

Figure 2 Recruitment flow chart. CHP, Concerto Health Program; FMG, Family Medicine Group.

2018-067/01-06-2018with all protocol modifications being mandatory to report (see online supplementary files 5 and 6). All participants will provide their informed consent following a procedure approved by the ethics board (see online supplementary files 7-9) before enrolment in the study. All data will be anonymised and will be used only for statistical research and analysis. They will be securely stored on the server of Canadian Research Chair on Technologies and Practices in Health, we will never share it with third parties. Only the principal investigator, the research coordinator and eventually students who work on the project will have access to the list of participants in the different phases of the project. Data from EMR will be also anonymised by a medical secretary or 
a research assistant who will sign a confidentiality agreement. In addition, all team members will sign a confidentiality agreement so that any personal information of participants will not be shared.

In keeping with our participative approach and inspired by frameworks of collaboration between researchers and knowledge users, ${ }^{63}{ }^{64}$ knowledge translation will be done in an integrated way throughout the project, with an emphasis on collaboration, shared outcomes and feedback from stakeholders at each step of the research. We will also share the outcomes through presentations in the networks and organisations of the team members, and through the production of dissemination tools for patient and citizen groups and associations. Ideally, these presentations will be done in tandem (patient-researcher; patient-clinician) in an interactive way, by taking the time for discussion and exchanges with the audience (eg, lunch and learn, scientific café). The presentations will be supported with materials (brief reports, narrated slideshows, etc) allowing a greater dissemination of the activities and outcomes. Knowledge translation activities at the end of project will consist of publishing outcomes in open access peer-reviewed journals. Presentations at national and international conferences in health informatics, chronic diseases and patient engagement are also scheduled.

\section{Study status}

This is an ongoing study taking place from December 2017 to December 2019. At the time of writing, the prototype of the eHealth technology module was designed and the first usability test was done.

\section{DISCUSSION}

This project shows a potential of success through the involvement of the technological partner who has a long collaborative experience with researchers. The eHealth solution is also likely to be acceptable because it will be adapted to patient's needs, based on our user-centred approach and the adaptation of the content to users' literacy level. Previous results associated with the use of the CHP solution for clinicians show promising preliminary outcomes based on validated measures that are relevant and sensitive to the proposed intervention. The solution has also a good potential for sustainability given that it will be implemented in the real context of primary care practice, with the collaboration of clinical teams. Finally, the project team is engaged in disseminating the results and pursuing the development and adaptation of the CONCERTO+ solutionin order to contribute to improving the health of people in Canada and internationally.

\section{Protocol version}

Version 1 (November 15 th 2018).

\section{Author affiliations}

${ }^{1}$ Centre de Recherche sur les Soins et les Services de Première Ligne de I'Université Laval, Quebec City, QC, Canada
${ }^{2}$ Faculty of Nursing Sciences, Université Laval, Quebec City, QC, Canada

${ }^{3}$ Groupe Santé Concerto, Montréal, QC, Canada

${ }^{4}$ Patient expert, Quebec City, QC, Canada

${ }^{5}$ Faculty of Medicine and Pharmacy, VrjeUniversiteit, Brussel, Belgium

${ }^{6}$ Department of Family and Emergency Medicine, Université Laval, Quebec City, QC, Canada

${ }^{7}$ Department of Health Management, Evaluation and Policy, School of Public Health, Université de Montréal, Montréal, QC, Canada

${ }^{8}$ École de design, Université Laval, Quebec City, QC, Canada

${ }^{9}$ School of Health Sciences (HESAV), HES-SO University of Applied Sciences and Arts Western Switzerland Lausanne, Lausanne, Switzerland

${ }^{10}$ Department of Family Medicine, Mc Gill University, Montréal, QC, Canada

Contributors $\mathrm{M}-\mathrm{PG}, \mathrm{AL}, \mathrm{GC}$ and $\mathrm{CC}$ conceived and designed the study and drafted the manuscript. AG, J-PF, FLég, HOW, AL, RB, CS, AM, SA-R, CD, EK, FLép, JR and MAN participated in designing the study and revised the manuscript. All authors read and approved the final manuscript.

Funding This study is funded by the Canadian Institutes of Health Research. Funding reference number: PCG-155469.

Competing interests None declared.

Patient consent for publication Not required.

Ethics approval This study has ethical approval from the sectorial committee of research ethics in health science of Université Laval. Approval number: 2018-067/01-06-2018

Provenance and peer review Not commissioned; peer reviewed for ethical and funding approval prior to submission.

Open access This is an open access article distributed in accordance with the Creative Commons Attribution Non Commercial (CC BY-NC 4.0) license, which permits others to distribute, remix, adapt, build upon this work non-commercially, and license their derivative works on different terms, provided the original work is properly cited, appropriate credit is given, any changes made indicated, and the use is non-commercial. See: http://creativecommons.org/licenses/by-nc/4.0/.

\section{REFERENCES}

1. World Health Organization. Global status report on noncommunicable diseases. 2014. http://apps.who.int/iris/bitstream/ handle/10665/148114/9789241564854_eng.pdf.

2. Roberts KC, Rao DP, Bennett TL, et al. Prevalence and patterns of chronic disease multimorbidity and associated determinants in Canada. Health Promot Chronic Dis Prev Can 2015;35:87-94.

3. Health Council of Canada. Helping patients help themselves: are Canadians with chronic conditions getting the Support they need to manage their health? 2010. https://healthcouncilcanada.ca/files/2. 17-AR1_HCC_Jan2010.pdf.

4. Ruel G. Multimorbidité: Ampleur, impacts et défi. 4e Rendez-vous des maladies chroniques. Quebec: Institut national de santé publique du Québec, 2012. (accessed 28 Apr 2017).

5. Fortin M, Contant E, Savard C, et al. Canadian guidelines for clinical practice: an analysis of their quality and relevance to the care of adults with comorbidity. BMC Fam Pract 2011;12:74.

6. Bounajm F, Dinh T, Thériault L. Améliorer les habitudes de vie: des retombées importantes pour la santé et l'économie du Québec. 2017. https://www.conferenceboard.ca/temp/7230eaa9-bb8f-43ae8ecb 969e71c8c696/6647_SanteEtEconomieDuQuebec_RPT.pdf (accessed 29 May 2018).

7. The Commissaire à la Santé et au Bien-Être. Adopting an integrated approach to chronic disease prevention and management: recommendations, issues and implications. 2010. https://www.csbe. gouv.qc.ca/fileadmin/www/2010/MaladiesChroniques/CSBE_T4_ Recommandations2010.pdf.

8. Ministère de la Santé et des Services Sociaux. Cadre de référence pour la prévention et la gestion des maladies chroniques physiques en première ligne. 2012. http://publications.msss.gouv.qc.ca/msss/ fichiers/2012/12-942-01F.pdf.

9. FMOQ, The Fédération des médecins omnipraticiens du Québec. Communiqué de la FMOQ: La FRMOQ lance une nouvelle campagne publicitaire pour rappeler l'urgence d'investir en médecine familiale, 2011. Montréal.

10. Bates-Eamer N, Ronson JL, shortage P. Perceived shortage, relative surplus: the paradox of Quebec's Family physician workforce - An 
Intra- and Inter-Provincial Comparison, U.o.V, Centre for Global Studies. Ed. 2009.

11. OCDE, The Organization for Economic Co-operation and Development: Promoting health, preventing disease, the economic case. The European Observatory on Health Systems and Policies Series. 2015. https://www.oecd-ilibrary.org/docserver/ 9780335262274-en. pdf?expires $=1535485524 \& i d=i d \& a c c n a m e=$ guest\&checksum =37E5F5C0D17CCC858CBF41B9E570C708 (Accessed 28 Aug 2018).

12. Adams SG, Smith PK, Allan PF, et al. Systematic review of the chronic care model in chronic obstructive pulmonary disease prevention and management. Arch Intern Med 2007;167:551-61.

13. Longtin $\mathrm{Y}, \mathrm{Sax} \mathrm{H}$, Leape LL, et al. Patient participation: current knowledge and applicability to patient safety. Mayo Clin Proc 2010;85:53-62.

14. Hibbard JH, Greene J. What the evidence shows about patient activation: better health outcomes and care experiences; fewer data on costs. Health Aff 2013;32:207-14.

15. Greene J, Hibbard JH. Why does patient activation matter? An examination of the relationships between patient activation and health-related outcomes. J Gen Intern Med 2012;27:520-6.

16. Hibbard JH, Mahoney ER, Stock R, et al. Do increases in patient activation result in improved self-management behaviors? Health Serv Res 2007;42:1443-63.

17. Fowles JB, Terry P, Xi M, et al. Measuring self-management of patients' and employees' health: further validation of the Patient Activation Measure (PAM) based on its relation to employee characteristics. Patient Educ Couns 2009;77:116-22.

18. Mosen DM, Schmittdiel J, Hibbard J, et al. Is patient activation associated with outcomes of care for adults with chronic conditions? J Ambul Care Manage 2007;30:21-9.

19. Becker ER, Roblin DW. Translating primary care practice climate into patient activation: the role of patient trust in physician. Med Care 2008;46:795-805.

20. Hibbard JH, Cunningham PJ. How engaged are consumers in their health and health care, and why does it matter? Res Brief 2008;8:1-9.

21. Remmers C, Hibbard J, Mosen DM, et al. Is patient activation associated with future health outcomes and healthcare utilization among patients with diabetes? J Ambul Care Manage 2009;32:320-7.

22. Donald M, Ware RS, Ozolins IZ, et al. The role of patient activation in frequent attendance at primary care: a population-based study of people with chronic disease. Patient Educ Couns 2011;83:217-21.

23. Greene J, Hibbard JH, Sacks $\mathrm{R}$, et al. When patient activation levels change, health outcomes and costs change, too. Health Aff 2015;34:431-7.

24. Holmström I, Röing M. The relation between patient-centeredness and patient empowerment: a discussion on concepts. Patient Educ Couns 2010;79:167-72.

25. Pomey M-P, Flora L, Karazivan P, et al. Le «Montreal model» : enjeux du partenariat relationnel entre patients et professionnels de la santé Santé Publique 2015;S1:41-50.

26. Kickbusch I, Pelikan J, Apfel F, et al. Health literacy. World Health Organisation. 2013. http://www.euro.who.int/_data/assets/pdf_file/ 0008/190655/e96854.pdf.

27. Hart JT. The Autonomous Patient: Ending Paternalism in Medical Care. JRSM 2002:95:623-4

28. Koh HK, Brach C, Harris LM, et al. A proposed 'health literate care model' would constitute a systems approach to improving patients engagement in care. Health Aff 2013:32:357-67.

29. Frosch DL, Elwyn G. Don't blame patients, engage them: transforming health systems to address health literacy. $J$ Health Commun 2014;19 Suppl 2:10-14.

30. Monkman $\mathrm{H}$, Andre W. eHealth literacy issues, constructs, models, and methods for health information technology design and evaluation. Knowledge Management \& E-Learning: An International Journal 2015;7:541-9.

31. Chaudhry B, Wang J, Wu S, et al. Systematic review: impact of health information technology on quality, efficiency, and costs of medical care. Ann Intern Med 2006;144:742-52.

32. Cummings $E$, Turner P. Patient self-management and chronic illness: evaluating outcomes and impacts of information technology. Stud Health Technol Inform 2009;143:229-34.

33. Dorr D, Bonner LM, Cohen AN, et al. Informatics systems to promote improved care for chronic illness: a literature review. J Am Med Inform Assoc 2007:14:156-63.

34. Häyrinen K, Saranto K, Nykänen P. Definition, structure, content, use and impacts of electronic health records: a review of the research literature. Int J Med Inform 2008;77:291-304.
35. Lau F, Kuziemsky C, Price M, et al. A review on systematic reviews of health information system studies. J Am Med Inform Assoc 2010;17:637-45.

36. Rigby M, Georgiou A, Hyppönen $\mathrm{H}$, et al. Patient Portals as a Means of Information and Communication Technology Support to Patient- Centric Care Coordination - the Missing Evidence and the Challenges of Evaluation. A joint contribution of IMIA WG EVAL and EFMI WG EVAL. Yearb Med Inform 2015;10:148-59.

37. Eland-de Kok P, van Os-Medendorp H, Vergouwe-Meijer A, et al. A systematic review of the effects of e-health on chronically ill patients. $J$ Clin Nurs 2011;20:2997-3010.

38. Irizarry T, DeVito Dabbs A, Curran CR. Patient portals and patient engagement: A state of the science Review. J Med Internet Res 2015;17:e148.

39. Price M, Bellwood P, Kitson N, et al. Conditions potentially sensitive to a personal health record (PHR) intervention, a systematic review. BMC Med Inform Decis Mak 2015;15:32.

40. Ross J, Stevenson F, Lau R, et al. Factors that influence the implementation of e-health: a systematic review of systematic reviews (an update). Implement Sci 2016;11:146.

41. Strisland F, Svagård. AH, Reitan J. Meeting end user needs in international research projects aiming to develop medical device technology prototypes for integrated care: A case study. Int J Integr Care 2016:16.

42. Monkman $\mathrm{H}$, Kushniruic $\mathrm{A}$. Applying usability methods to identify health literacy issues: an example using a Personal Health Record. Stud Health Technol Inform 2013;183:179-85.

43. Research2Guidance. conomics: The current status and trends of the mHealth app market. 2017. https://www.uzelf.org/wp-content/ uploads/2017/12/R2G-mHealth-Developer-Economics-2017-StatusAnd-Trends.pdf.

44. Greenhalgh T, Hinder S, Stramer K, et al. non-adoption, and abandonment of a personal electronic health record: case study of HealthSpace. BMJ 2010;341:c5814.

45. Garabedian LF, Ross-Degnan D, Wharam JF. Mobile phone and smartphone technologies for diabetes care and self-management. Current diabetes reports 2015;15:109.

46. Group N. Catalyst insights council monthly report: How to hardwire patient engagement into care delivery processes. 2017. https:// join.catalyst.nejm.org/hubfs/lnsights\%20Council\%20Monthly\% 20-\%20Files/Insights\%20Council\%20Monthly\%20May\%202017\% 20Report_How\%20to\%20Hardwire\%20Patient\%20Engagement\% 20into\%20Care\%20Delivery\%20Processes.pdf.

47. Khoja S, Durrani H, Scott RE, et al. Conceptual framework for development of comprehensive e-health evaluation tool. Telemedicine and e-Health 2013;19:48-53.

48. Lawani MA, Valéra B, Fortier-Brochu É, et al. Five shared decisionmaking tools in 5 months: use of rapid reviews to develop decision boxes for seniors living with dementia and their caregivers. Syst Rev 2017;6:56.

49. Zanaboni P, Ngangue P, Mbemba GIC, et al. Methods to Evaluate the Effects of Internet-Based Digital Health Interventions for Citizens: Systematic Review of Reviews. JMIR 2018;20:e10202.

50. Giguere AM, Labrecque M, Haynes RB, et al. Evidence summaries (decision boxes) to prepare clinicians for shared decision-making with patients: a mixed methods implementation study. Implement Sci 2014:9:144

51. Lemieux V. Pour qu'on se comprenne: guide de littératie en santé. 2014. https://publications.santemontreal.qc.ca/uploads/tx asssmpublications/litteratie_v9.pdf.

52. Hibbard JH, Mahoney ER, Stockard J, et al. Development and testing of a short form of the patient activation measure. Health Services Research 2005;40:1918-30.

53. Health I. Patient Activation Measure. 2014. http://www.insigniahealth $\mathrm{com} /$

54. Glasgow RE, Wagner EH, Schaefer J, et al. Development and validation of the patient assessment of chronic illness care (PACIC). Medical care 2005:436-44.

55. Davis FD, usefulness P. perceived ease of use, and user acceptance of information technology. MIS quarterly 1989:319-40.

56. Solomon M, Wagner SL, Goes J. Effects of a Web-based intervention for adults with chronic conditions on patient activation: online randomized controlled trial. JMIR 2012;14:e32.

57. Ghandour E, Gagnon M-P, Fortin J-P. Patient adoption of a connected electronic personal health record to support chronic disease management in primary care in Quebec. Submitted for publication.

58. Ghandour E, Gagnon M-P, Fortin J-P. Conditions d'adoption du dossier de santé électronique personnel par les professionnels de la première ligne au Québec: Perspectives professionnelle et organisationnelle. Revue Santé Publique 2017;29:837-50. 
59. World Health Organization. Beginning with the end in mind planning pilot projects and other programmatic research for successful scaling up. 2014. https://www.who.int/reproductivehealth/publications/ strategic approach/9789241502320/en/.

60. Hendriks M, Rademakers J. Relationships between patient activation, disease-specific knowledge and health outcomes among people with diabetes; a survey study. BMC health serv res 2014; 14:393.

61. Aronson J. A pragmatic view of thematic analysis. Qualitative Report 1994;2:1-3.
62. Miles MB, Huberman AM. Qualitative data analysis: An Expanded Sourcebook. Sage 2003.

63. Parry D, Salsberg J, Macaulay AC, et al. A Guide to Researcher and Knowledge-User Collaboration in Health Research. C.I.o.H.R. (CIHR) Editor 2009:83.

64. Baumbusch JL, Kirkham SR, Khan KB, et al. Pursuing common agendas: a collaborative model for knowledge translation between research and practice in clinical settings. Res Nurs Health 2008;31:130-40. 